\title{
Mathematical Intuition and Natural Numbers: A Critical Discussion
}

\section{Charles Parsons, Mathematical Thought and Its Objects, Cambridge University Press, New York, 2008, xx + 378 pp}

\author{
Felix Mühlhölzer
}

Received: 12 November 2009/ Accepted: 2 March 2010/Published online: 5 June 2010

(C) The Author(s) 2010. This article is published with open access at Springerlink.com

\begin{abstract}
Charles Parsons' book "Mathematical Thought and Its Objects" of 2008 (Cambridge University Press, New York) is critically discussed by concentrating on one of Parsons' main themes: the role of intuition in our understanding of arithmetic ("intuition" in the specific sense of Kant and Hilbert). Parsons argues for a version of structuralism which is restricted by the condition that some paradigmatic structure should be presented that makes clear the actual existence of structures of the necessary sort. Parsons' paradigmatic structure is the so-called 'intuitive model' of arithmetic realized by Hilbert's strings of strokes. This paper argues that Hilbert's strings, considered as given in intuition, cannot play the role Parsons assigns to them: the criteria of identity of these strings do not have the sharpness that Parsons wants to see in them, and Parsons inadvertently projects abstract structures into his 'intuitive model'. This diagnosis is exemplified with respect to (a) Parsons' distinction between addition and multiplication on the one hand and exponentiation on the other and (b) his analysis of arithmetical knowledge in simple cases like " $7+5$ $=12$ ". All in all, it is claimed that Parsons book contains many important insights with respect to, for example, different versions structuralism, the notion of "natural number" and its uniqueness, induction, predicativity and other things, for which he is rightly famous, but that his way of drawing on the notion of intuition leaves too many questions unanswered.
\end{abstract}

\section{Some General Remarks}

Mathematical Thought and Its Objects is the fruit of many years of scrupulous thinking about two topics in the philosophy of mathematics, which Parsons himself identifies as the book's main themes: first, the notion of object as it is deployed in

F. Mühlhölzer (ه)

Georg-August-Universität, Göttingen, Germany

e-mail: fmuehlh@gwdg.de 
mathematics, with special emphasis on natural numbers as the most prominent objects; and second, a particular notion of mathematical intuition emanating from conceptions of 'Anschauung' to be found in the work of Kant and Hilbert. Much of the material of this book has been presented in articles from 1980 to 2004, and the chapters roughly correspond to these articles; but Parsons has added amendments and new defenses, answering criticisms leveled since then. In spite of this updating, the different dates of the parts of the text remain noticeable, and Parsons' complicated trains of thought are very often interrupted, readopted at later points, interrupted again, and so on, which makes the reading of his book not an easy-going affair. Furthermore, Parsons' extreme scrupulousness has the effect that rather often the results of his thoughts appear to be left in abeyance, and even the two most important characteristics of his philosophical position, his specific version of structuralism and the precise role of 'intuition' within this version, are difficult to identify. In the following critical discussion I shall be mainly occupied with these two issues, but before some more general remarks are in order.

First, the reader of Parsons' book should be warned that its title is rather misleading. The 'mathematical thought' discussed in it is not the thought of practicing mathematicians working in central areas of mathematics, but only the thought to be found in mathematical logic, in very elementary arithmetic, in proof theory and in set theory. Accordingly, also the domain of 'mathematical objects' considered by Parsons is extremely restricted, ultimately to natural numbers (sets are considered as well, but mainly with regard to set-theoretic analyses of natural numbers) and to certain 'intuitively given' proxies of natural numbers, like Hilbert's strings of " 1 "s or of strokes. It is a long-standing trademark of Parsons' philosophizing to show an impressive competence with respect to the technical subtleties of the areas treated by him, but in his restriction to areas that make up only a very small and, from the point of view of many working mathematicians, marginal part of real mathematics, Parsons' philosophy suffers from an unbalanced diet.

Of course, this restriction is typical of 'orthodox' philosophy of mathematics, but it contradicts Parsons' own claim to give a "more descriptive approach to mathematical knowledge" (xiv-xv). Parsons has a healthy tendency to resist metaphysical ambitions and to replace them by linguistic considerations or by genetic narratives, most prominently in the case of the genesis of our concept of natural number, but the one-sidedness of his orthodox point of view leads to characteristic distortions. This is particularly clear on pp. 109-10, where Parsons takes up Paul Bernays' important thought that reference to mathematical objects is relative to a background structure, which, however, can be understood in two different ways:

The first idea is that the objects referred to all fit into a single comprehensive structure of which the most obvious example would be sets with the membership relation. The other is that we might have a number of different structures that provide "homes" for objects of different kinds. The second fits better with the language of informal mathematics, before logicians undertake 
to formalize it. It also provides a good setting for discussing the issues that arise for structuralist views. (p. 110)

It is characteristic of Parsons' style that, in spite of what he himself sees as the advantages of this second idea, he at this point pleads for adoption of the first. He simply bows here to the judgment of a set theorist who observed that "it may be necessary to make connections with ostensibly remote parts of mathematics in order to arrive at a proof" (p. 110) und who claimed that set theory "is the theory that has been developed most fully for this purpose and remains the leading candidate for the role of framework for all of mathematics" (p. 110). Now, Andrew Wiles, say, whose proof of Fermat's Theorem is a paradigm of a proof connecting remote parts of mathematics, would be astonished to hear that it is set theory which accomplishes this task. Obviously, Parsons is moving too far away from real mathematics here. ${ }^{1}$ Furthermore, Parsons considers Bernays' idea as an essential part of a structuralist view of mathematical objects, ${ }^{2}$ and in the way he himself then develops this view, set theory does not seem to be the supposed background structure. So Parsons actually knows better than suggested in the passage just cited.

Parsons" main theme is "mathematical intuition" rather than "mathematical thought". Even in Chap. 9, which is entitled "Reason", the main topic turns out to be "intuition", namely "rational intuition" in contrast to the Kant-Hilbertian intuition discussed in the chapters before. In the way he gives 'intuition' a prominent place in his philosophy, Parsons is neither a naturalist nor a nominalist. Against Hartry Field's nominalistic physicalism, for example, which makes the justification of mathematics dependent upon hypotheses about the physical world, he plausibly argues that this view is "more vulnerable to refutation than the mathematics [which it wants to adjust to physicalist prejudices]" (p. 65). Nevertheless, Parsons takes Field's view very seriously and spends a lot of time criticizing it; but since it is contrary to his own way of thinking, this criticism remains halfhearted and will not convince anybody with strong tendencies towards nominalism. In accordance with Parsons' descriptive approach, it would have been more appropriate to react like Tyler Burge, who simply declares such nominalist efforts "not to be worth tilting with" (Burge 2003, p. 214, note 22).

In his attempt to rescue a notion of "intuition of objects", that constitutes a separate epistemic category besides "understanding" and "reason", and in pursuing the question of "how much of arithmetic is intuitively known" (xii), Parsons sees his philosophy of mathematics as belonging to the Kantian tradition. For good

\footnotetext{
${ }^{1}$ In an illuminating letter to his sister Simone (Weil 1940), the mathematician André Weil emphasized the importance of analogies in mathematics, with analogies between algebraic functions and numbers as his main example, and at the end he develops the Bourbakian thought that such analogies may be expressed axiomatically and in this way contribute to the unification of mathematics: "a unification, which absorbs in some simple and general theories all the common substrata of the diverse branches of the science, suppressing what is not so useful and necessary, and leaving intact what is truly the specific detail of each big problem" (Weil 1940, p. 341). This sophisticated idea of unification is very different from the simple-minded idea of a reduction of mathematics to set theory which obviously abounds in things that are "not so useful and necessary".

2 "By [a 'structuralist view' of mathematical objects] I mean the view that reference to mathematical objects is always in the context of some background structure, and that the objects involved have no more by way of a 'nature' than is given by the basic relations of the structure" (p. 40).
} 
reasons, Parsons' Kantianism, however, is a considerably softened one, because his Quinean heritage prevents him from finding a respectable place for the a priori; and his orientation towards Hilbert's 'finitary attitude' leads him to a far-reaching attenuation of Kant's notion of intuition ("Anschauung") by applying it, like Hilbert, mainly to signs regarded as geometric shapes. For Parsons these signs are what he calls "quasi-concrete objects", and with this theoretical notion, which we will examine later, and with the specific use he makes of it, Parsons abandons the descriptive approach in his philosophizing and indulges in philosophical theorizing reminiscent of Kant. We have to understand, then, what motivates this philosophical move and whether it is successful.

Parsons' book is too rich to be reviewed exhaustively. This critical discussion will be mainly concerned with Parsons' specific version of structuralism and with the role he ascribes to 'intuition'. ${ }^{3}$ I will be rather critical of Parsons' position, but would like to emphasize that this is owing to the one-sidedness of my perspective, which makes me leave out many of the book's virtues. So I do not convey anything of the richness and scrupulousness of Parsons' discussion of the many different possible brands of structuralism, from which he then distances himself with his own version. I almost completely ignore his profound reflections on set theory, and my concentration on the topic "intuition" even makes me leave out (with a very heavy heart, I should say) many of his differentiated thoughts about natural numbers. According to Parsons, natural numbers are not intuitively given, despite the fact that we can have intuitive knowledge about some elementary arithmetical facts (via intuitively given instances of the structure of natural numbers). Therefore, also the many non-elementary facts about natural numbers that are inaccessible to intuitive knowledge will not be taken into account in the present discussion. This abstinence concerns Parsons' profound reflections on induction and on predicativity, and it concerns his original treatment of the issue of the uniqueness (up to isomorphism) of the natural number structure by considering the communication and mutual understanding of mathematicians. All that will remain unconsidered here.

Furthermore, before beginning my detailed critique, I want to add that I find Parsons' way of philosophizing very impressive: he always paints with an extremely fine brush and thereby shows a seriousness and an anti-narcissism which are without equal. Everybody who goes to the time and effort of reading this book will ingest an unforgettable model of really honest philosophy. At the same time, however, I do not want to conceal the fact that Parsons' extreme seriousness also has its dark side, because it seems to tie him to old, familiar paths and to prevent him from unlocking new ground. For example, one might be impressed at first sight when reading, in the context of the topic "structuralism", the frank confession on p. 109: "I have to leave category theory out of account because of insufficient knowledge"; but when following Parsons' further considerations, one begins to be sorry that he had not acquainted himself with category theory. It would have been so helpful for his purposes! As described, for example, in Barry Mazur's beautiful article "When is

\footnotetext{
3 A helpful critical commentary on every section of Parsons' book has been written by Peter Smith (2009) and a succinct overview by John Burgess (2008). Smith and Burgess, however, admit that they feel uneasy about Parsons' emphasis on "intuition", and they deal with this topic in a relatively superficial way. In contrast to this, I will bring "intuition" into focus.
} 
one thing equal to some other thing?", the structuralist perspective which category theory opens up with regard to 'mathematical objects' and in particular to natural numbers, permits a very natural representation of the characteristic 'uniqueness up to isomorphism' of mathematical objects and offers

a grand view of the nature of mathematical objects that has taken root in mathematical culture during the past half-century, [.... In order to appreciate it] we must [...] become conversant with a language that has a thrust somewhat different from the standard fare of foundations. This newer vocabulary has [...] something to say about every part of mathematics, including the definition of the natural numbers. (Mazur 2007, p. 4)

Parsons' style of philosophizing relies strongly on technical achievements. The fact that he remains within the narrow range of orthodox foundations which belong to the time prior to the past half-century, and that he does not even take a look at the achievements of category theory, can be felt as a severe gap in his book.

\section{Parsons' Structuralism}

In Chapter 1 ("Objects and logic") Parsons presents and defends the view that "[s]peaking of objects just is using the linguistic devices of singular terms, predicates, identity, and quantification to make serious statements" (p. 10)—nothing more is demanded. He sums this up as the view "that the most general notion of object has its home in formal logic" (p. 10), which sounds all too narrow if with the term "formal logic" one associates formal languages with rigorous rules of formation and derivation, but, if I understand him correctly, Parsons' doesn't mean it in this sense. On p. 7 he explains that "formal logic" is used here in the Kantian sense, referring to "the forms of judgments rather than the categories", which allows 'forms' belonging also to non-formal languages. This liberal notion of object is particularly appropriate in the case of mathematical objects, and it fits especially well with a structuralist view of them, which Parsons wants to develop.

He starts from the, as it were, classical statement of this view by Michael Resnik:

In mathematics [...] we do not have objects with an "internal" composition arranged in structures, we have only structures. The objects of mathematics, that is, the entities which our mathematical constants and quantifiers denote, are structureless points or positions in structures. As positions in structures, they have no identity or features outside of a structure. (Resnik 1981, p. 530)

One might expect that, in accordance with his liberal notion of an object, Parsons would make use of this idea by simply identifying mathematical objects with 'positions in structures', or maybe with the 'roles' they play within structures, but (if I got it right) he rejects this philosophical move, presumably because he rejects the metaphysical pictures underlying this sort of talk.

Before discussing the details of Parsons' own version of structuralism, I would like to point out one characteristic defect that it shares with many other structuralist positions and which may be typical of the structuralist approach as such: it consists 
in the wide neglect of mathematical proofs. In fact, the topic "proof" plays only a minuscule part in Parsons' considerations. Even the Chap. 8 about mathematical induction is mainly concerned with the issue of the adequate formulation and understanding of the principle of induction, with regard to the role this principle plays for our understanding of the concept of natural numbers, and not with inductive proofs as such. Also Parsons' search for an adequate role for 'intuition' in mathematics is much more concerned with intuition of objects than with aspects of proof that might be called 'intuitive'. ${ }^{4}$ Parsons' far-reaching neglect of proofs is a further reason for the inadequacy of his book's title, because obviously 'mathematical thought' is mainly deposited in proofs.

Parsons, however, has good reasons for his claim to present a version of structuralism that is preferable to other ones, and especially preferable to what he calls eliminative structuralism. He devotes more than 50 pages (pp. 46-100) to a penetrating critique of eliminative structuralists, whose main idea consists in 'eliminating' the reference to objects that are considered as 'not respectable'-from a nominalistic or physicalistic perspective, say-by accepting only structures realized by respectable ones. Take the example of natural numbers, considered as not respectable. Let $A(N, O, S)$ be an ordinary (albeit regimented) statement about natural numbers, with names " $N$ ", " $O$ " and " $S$ ", by which, in a philosophically noncritical mood, we think of "the set of natural numbers", "the initial element of the progression of natural numbers" and "the successor function on the set of natural numbers", respectively. We then give an axiomatization (preferably a categorical one) of the 'structure of natural numbers', expressed by an open sentence $\Omega(N, O, S)$ with variables " $N$ ", " $O$ " and " $S$ " (again in such a way that, when being in a philosophically non-critical mood, we think of "the set of natural numbers", "the initial element of the progression of natural numbers" and "the successor function on the set of natural numbers"), and we then treat what we wanted to say by " $A(N$, $0, S)$ " as elliptical for

(E) For any $N, O, S:$ if $\Omega(N, O, S)$, then $A(N, O, S)$.

The point of this reconstruction is that we now can interpret the quantifiers as ranging only over respectable objects. These may include respectable sets of objects, as suggested by the explication just given; but we may interpret (E) also as a second-order statement; or we use plural quantification; or, in order to enhance its plausibility and capability, we interpret (E) as quantifying not merely over actual objects but over possible ones. Thus, there are quite a lot of different versions of eliminative structuralism, and Parsons deals with all of them.

\footnotetext{
${ }^{4}$ In this respect, despite Parsons' reference to Kant and Brouwer in his Preface (xi-xii), his approach is very different from theirs, which relies on the idea of proof as construction, and Parsons himself registers this difference (see fn. 36 on p. 334). At this point one can even see a strong contrast to Hilbert's presentation of his idea of a finitary arithmetic and of proof theory. Already Hilbert's first text presenting this program (Hilbert 1922) contains the sketch of a real proof of the commutativity of addition (see paragraph 30 of Hilbert 1922). It is an intuitive version of a proof by infinite descent (which is a variant of proofs by induction), and intuitive proofs of this sort would certainly deserve an in-depth discussion in investigations of the role of intuition in mathematics.
} 
People who have no qualms about the respectability of natural numbers will not find eliminative structuralism concerning natural numbers attractive from the outset, the more so as it has an obvious problem: if there are no respectable candidates of $N$, $O$ and $S$ making $\Omega(N, O, S)$ true-and from the point of view of nominalism or physicalism, for example, the existence of such candidates is certainly anything but self-evident-(E) will be vacuously true which, of course, it should not be. Parsons calls this the problem of nonvacuity (p. 49), and according to him it concerns eliminative structuralism in its entirety. He thinks that this problem is obviously unsolvable in the eliminative versions of higher set theory; he dismisses the nominalistic and physicalistic prejudices motivating many versions of eliminative structuralism; and he criticizes the lack of "faithfulness to mathematical discourse" (p. 94) shown by rational reconstructions in the manner of (E). Since I share Parsons' attitude in all these respects, I will not discuss his critique in more detail. ${ }^{5}$

To criticize eliminative structuralism on the ground of its lack of faithfulness to mathematical discourse can only be convincing if one offers a descriptively more adequate position, and this is Parsons' aim in developing his own, noneliminative version of structuralism (which is the topic of $\S 18$, the longest section of his book). It lives on the idea that we "can take the language of mathematics more at face value" and that we do not "take more as objectively determined about the objects about which it speaks than that language itself specifies" (p. 100). It is noneliminative in defending the idea of having "genuine reference to objects [even] if the »objects« are impoverished in the way in which elements of mathematical structures appear to be" (p. 107). Unfortunately, however, Parsons does not give an easily graspable presentation of his view, ${ }^{6}$ and I will not make the attempt to rationally reconstruct it in a coherent way. Instead, I simply want to highlight some of its most important characteristics.

Let us start with Parsons' very interesting — and, as it seems to me, profoundhandling of the notion of a 'structure'. There is the notorious problem of structuralism that if we want to give a non-circular structuralist account of sets, we had better not use set theory itself in order to explain what a 'structure' is. In Parsons' more precise description:

We want to talk of structures for set theory without supposing that their domains are sets. Also, the set-theoretic conception [of structure] requires a decision about the ontology of set theory itself (say, in favor of ZF rather than a von Neumann-style formulation), which the most fundamental conception of structure should not make. (p. 114)

Parsons' solution to this problem consists in adopting what he calls a metalinguistic notion (or conception) of structure which, in first approximation, simply says that the domain of a structure is given by a suitable predicate and the relations and functions by further predicates and functors (pp. 111-112). The application of this idea to sets is then described as follows:

\footnotetext{
5 Such a discussion can be found in Smith 2009, pp. 7-14.

6 Burgess states that Parsons' "own version of structuralism is only rather sketchily indicated" (Burgess 2008, p. 40), and similar complaints can be found in Smith 2009.
} 
We can define "structures" to interpret the language of set theory by using our mathematical vocabulary, including the predicates ' () is a set' and ' () is an element of []'. Without such vocabulary, or other mathematical vocabulary of similar abstraction, we will not be able to describe a structure satisfying the axioms of set theory; that is the "bootstrapping" aspect of the understanding of set theory. But otherwise, the metalinguistic conception of structure works in the same way as it does in the more elementary cases. (pp. 113-114)

The 'more elementary cases' would be cases in which the predicates and functors are considered as antecedently well understood, as this is paradigmatically the case for Hilbert's finitary arithmetic, for example, where the objects are strings of strokes-i.e. are given by the predicate '( $)$ is a string of strokes'—and the relations and functions have simple intuitive meanings. In the non-elementary case of set theory, though, we need a 'bootstrapping' approach which lets us understand the predicates ' () is a set' and ' () is an element of []' more holistically by their connections to other notions, including more elementary ones. This perspective makes it possible for Parsons to defend in Chap. 4 ("A problem about sets") a structuralist account of set theory that appears as a reasonable minimal position avoiding the defects of alternative views. Since I find this account plausible, I will not discuss it in more detail.

In accordance with the 'logical notion of object' explained in Chap. 1, Parsons regards a structuralist approach to mathematical objects based on a metalinguistic conception of structure as "the most elementary way of describing a kind of mathematical object [...]" (p. 114). But now, within this metalinguistic orientation, the topic "intuition" immediately comes to the fore, because this orientation involves an appeal to linguistic objects such as predicates and functors, which Parsons treats as expression types. As such they are objects which can be given to us by intuition and of which we can have intuitive knowledge. Furthermore, they are realized by concrete tokens, to which they stand in an internal relation (i.e., they are what Parsons calls "quasi-concrete objects" - a notion which will occupy us later), and in that way they defy structuralist views of objects, because these tokens do not belong to the structure in which the types may reside. This, of course, is a serious problem for structuralism if we want to defend a structuralist view not only in case of mathematical theories that are about numbers and similar purely abstract objects, but also in case of theories, like metamathematics, that, as often conceived, are about linguistic types. At this point, Parsons is ready to sacrifice his structuralism by admitting that linguistic types are an exception: they can be mathematical objects for which the structuralist view does not hold.

Parsons very well sees the objection that linguistic objects could be conceived also structuralistically, but he doesn't regard it as compelling. This objection says that a mathematical theory of linguistic objects, like theories in metamathematics, should by no means be considered in such a way that, for example, when dealing with Peano arithmetic, it refers to letters like "N" and "S". For these letters, with their characteristic, perceivable shapes, are, of course, totally arbitrary; we could just as well use "§" and "\%", etc. It is rather the role of these letters in the axiom system which is relevant. Let us call the linguistic objects identified with such a 
role, i.e. conceived as invariant when only their shape is changed but the role remains the same, formal symbols. ${ }^{7}$ Then one can easily see that the identity of these formal symbols is determined by the metatheory alone. Its identity doesn't depend on anything outside of the structures that the metatheory specifies, and so the structuralist view of mathematical objects can be maintained. As emphasized in Friederich 2010, everybody who does metamathematics with a minimum of understanding conceives of the linguistic objects referred to by a metamathematical theory in precisely this way. Obviously, this is a way in which the perceivable shape of the letters we use when actually writing down formulae in Peano arithmetic - and therefore our intuition of linguistic objects-is of no mathematical concern. ${ }^{8}$

For Parsons, however, this cannot be the whole story. He sticks to the view that structuralism has its limits in case of quasi-concrete objects like linguistic objects given in intuition. This is the reason he presents on p. 116 (similarly in fn. 58 on p. 168):

The more concrete domains, often of quasi-concrete objects, still play an ineliminable role in the explanation and motivation of mathematical concepts and theories. In particular, this is true of any mathematical treatment of formalized or natural languages. Thus, if the structuralist view of mathematical objects is taken to mean that all mathematical objects are only structurally determined, it has to rest on legislation about what counts as a mathematical object. The explanatory and justificatory role of more concrete models implies, in my view, that it is not the right legislation even for the interpretation of modern mathematics.

Certainly, the role of these more concrete objects, domains and models suggested in this programmatic passage should then be elaborated in detail. As admitted by Parsons himself, it cannot be important in order to give an adequate account of metamathematics in particular, and one wonders at this point what it precisely might be.

Both the rejection of eliminative structuralism and his adoption of a metalinguistic notion of structure belong to Parsons' "taking the language of mathematics at face value" (p. 100), which is one of the main points of his structuralism. What does he mean when he adds that this point "does not require us to take more as objectively determined about the objects about which [the language of mathematics] speaks than that language itself specifies" (p. 100)?-If one presupposes that the language of mathematics is speaking in the form of axiomatic systems, and since

\footnotetext{
7 I borrow this term from Friederich 2010 (who, moreover, reserves the term "linguistic object" to quasiconcrete objects only). In this paper, Simon Friederich shows that structuralism faces no particular problems with respect to metamathematics by arguing that from the outset metamathematics is not about quasi-concrete objects but about formal symbols. See also the technical elaboration of such an approach in Grzegorczyk 2005.

8 This perspective, by the way, makes up one important aspect of Wittgenstein's provocative statement, forming the title of a chapter of his Big Typescript, "There is no Metamathematics" (Wittgenstein 2005, p. 539). According to Wittgenstein, there is no metamathematics if metamathematics is conceived of as mathematics being about linguistic objects given in intuition, because the identity of mathematical objects is only determined by mathematics itself, which is not true, however, of objects given in intuition. See Mühlhölzer 2010 for an elaboration of this Wittgensteinian attitude.
} 
axiomatic systems at best determine the structures satisfying them only up to isomorphism, it follows that, strictly speaking, talking about 'the natural numbers' is not allowed. Unfortunately, Parsons sometimes seems to leave it open whether he accepts this consequence. This is what he says on p. 101:

[The structuralist view] seems to imply that if an isomorphic copy of the natural numbers can be constructed in another structure, then it is a question whether the copy is not just as good as the original, an equal claimant to be the natural numbers. Some copies do not have a good claim, such as the progressions constructed within the natural numbers or in some other way that obviously presupposes the natural numbers. But others are not so easy to rule out, the most prominent being the number sequences constructed in set theory and higher-order logic.

I am not sure what to make of the phrase "seems to imply" at the beginning of this passage. If one understands it in such a way that, even though reluctantly, Parsons has a tendency to accept said implication (apart from the cases where the progression is 'constructed within the natural numbers', which Parsons rules out), this would have consequences for what he calls the "sui generis view of the natural numbers" (pp. 101-103). As characterized on p. 101, this view states that natural numbers are not certain sets, or Fregean logical objects, or other entities acting as natural numbers. In other words, the natural numbers are not reduced to entities which are antecedently given and understood; they simply "are just what they are" (p. 101). To say this, however, means to tell a further story besides the structuralist one, and therefore a consequent structuralist should reject the sui generis view of natural numbers.

Parsons presents this result on p. 101, and if I understand him correctly, he adopts it. ${ }^{9}$ But what precisely are his reasons? After all, nobody has to be a consequent structuralist, and to make an exception to structuralism in elementary cases like the case of the natural numbers is by no means an implausible philosophical moveprobably not more so than taking the case of linguistic objects (considered as quasiconcrete objects) as such exceptions, as Parsons himself is arguing for. One may sense a clear argument against the sui generis view of natural numbers on p. 103, when Parsons considers equations like " $2=\{\varnothing,\{\varnothing\}\}$ " in set theory which, according to this view, appear to be blatantly false on the ground that 2 is not a set of this sort-where, however, these respectable equations certainly should not come out false. But this is an obviously silly argument, and Parsons immediately rejects it, because the symbol " 2 " is used in this set theoretic context simply as an abbreviation of " $\{\varnothing,\{\varnothing\}\}$ ", and according to the sui generis view, of course, the set $\{\varnothing,\{\varnothing\}\}$ is nothing but a set-theoretic ersatz for the genuine natural number 2 .

Parsons, though, takes such set theoretic reductions quite seriously in so far as they lead to what is called the multiple reductions problem, which arises from the fact that besides the von Neumann reduction of natural numbers, leading to the

\footnotetext{
${ }^{9}$ I infer this from the following statement on p. 101: "To see how our view of the natural numbers is structuralist, it is useful to consider another view [my emphasis] that is sometimes advanced, according to which natural numbers are sui generis".
} 
equation " $2=\{\varnothing,\{\varnothing\}\}$ ", there are also other ones like Zermelo's, for example, which gives rise to another equation of this sort, namely " $2=\{\{\varnothing\}\} "$. The supposed problem here consists in the question which of these reductions is 'the correct' one, and if there is no one 'correct' one, what to say then. On pp. 102-105, Parsons presents a complicated discussion of this issue, considering different possible ways of dealing with it, but in the end it has not become sufficiently clear to me what his own solution of the multiple relations problem is, or even whether he wants to give such a solution in the first place. My impression is that, though he cannot really bring himself to adopt it, at least at this point of the discussion he has a strong tendency to accept the sui generis view, which, as just described, seems to allow some sort of contextual analysis of equations like " $2=\{\varnothing,\{\varnothing\}\}$ " and " $2=$ $\{\{\varnothing\}\} "$ by saying that in different contexts one has the right to present different surrogates of the natural numbers, as long as the relevant structural properties of the domain of natural numbers are respected. Unfortunately, I am by no means sure of having really understood all of Parsons' considerations, and I would wish for a more resolute presentation of the reasons for his own view at this point of his reflections.

Up to now, in our discussion of Parsons' noneliminative structuralism there is still an important lacuna. It concerns the problem of nonvacuity, i.e. the issue concerning the existence of an instance of a structure, and therewith also of the objects residing in a structure. We have already seen that this is an essential problem for eliminative structuralism - but if I understand Parsons correctly, he thinks that it is a big problem for structuralism tout court, ${ }^{10}$ and therefore also for his own version. I find this astonishing, for his metalinguistic notion of structure seems to open a straightforward and well-known way out. It is Hilbert's way against Frege when Hilbert (in his letter to Frege of December 29, 1899) declares the consistency of an axiomatic system as the only criterion of the truth of its sentences and the existence of the objects referred to; and it is the way inherent in the position of Leitgeb and Ladyman 2008, approvingly mentioned by Parsons' himself on p. 108f. Leitgeb and Ladyman replace Hilbert's "consistency" by the more flexible talk about coherence, but this does not alter the philosophical strategy, which simply consists in shifting the problem of existence to the problem of coherence (a strategy that is well known from Quine's work). Furthermore, coherence is here something that concerns the actual language and practice of mathematics and that should not disquiet somebody like Parsons, who has adopted a metalinguistic orientation. And in fact Parsons himself uses precisely this strategy when he diverts scruples concerning the existence of structures of set theory in the following way: "We are able to understand higher set theory and to have enough of an intuition concerning the principles of set theory to create a highly developed theory, which shows no sign of being inconsistent or incoherent, and about which there is too much agreement for it to be ad hoc" (p. 99)—which, all in all, is enough to claim the existence of "the kind of structure higher set theory requires" (p. 99).

\footnotetext{
10 "However, the problem of nonvacuity, roughly that of the existence of an instance of the structure, is a recurring one in structuralist treatments of objects of different kinds, and will continue to occupy us" (p. 49).
} 
For Parsons, however, this cannot be the whole story. ${ }^{11}$ In his answers to 5 Questions (Hendricks and Leitgeb 2008) about his views on the philosophy of mathematics, he ends with the following statement: "Structuralists have said that the objects of the structure exist if the theory is coherent. But a lot more needs to be said about what that means than is said in my own writings or in the literature that I know" (Parsons 2008, p. 210). So in the present state of the discussion about these matters Parsons seems to judge a reliance on 'coherence' as nothing but handwaving. In Mathematical Thought and Its Objects he emphasizes the necessity of accommodating mathematical intuition, and this leads him to a "picture of number theory according to which the nonvacuity of the conception of natural numbers is made out by an example in which the objects are quasi-concrete" (p. 113). The example alluded to is the Hilbertian structure of strings of strokes, which will be discussed in the following section. Thus, Parsons here resorts to objects given in intuition in order to solve the problem of nonvacuity in the case of natural numbers: for him, the intuitively-given Hilbertian strings of strokes present an existing instance of a structure representing the natural numbers, i.e., they instantiate, as Parsons puts it, an intuitive model for the natural numbers. By accepting such objects of intuition as mathematical objects, Parsons leaves a thoroughgoing structuralist standpoint in the philosophy of mathematics, but he considers this "an unavoidable impurity in structuralism" (p. 219). ${ }^{12}$

This is precisely the point in Parsons' book that makes an in-depth discussion of his use of the notion of "intuition" unavoidable. In Kant's thinking, which is Parsons' starting point, "intuition" ("Anschauung") has an astonishingly broad spectrum of important functions; it is a fruitful theoretical term of Kant's philosophy. So, for example (and I am not trying to give a complete survey), "intuition" in Kant's work is meant in the sense of intuition of objects by which individual objects are given to our thinking. Intuition thereby gets the further important function of restricting our pure reasoning in order to prevent it from running into antinomies. And as pure intuition it explains the specific apriority of mathematics. The intuitionists and Hilbert in a sense retain the first two functions,

\footnotetext{
11 The mathematician Timothy Gowers, a Fields medalist and at the same time an adherent of the philosophical outlook of the later Wittgenstein, is less scrupulous. This is what he says in his nice little book Mathematics: A Very Short Introduction: "[T]here certainly are philosophers who take seriously the question of whether numbers exist, and this distinguishes them from mathematicians, who either find it obvious that numbers exist or do not understand what is being asked." (Gowers 2002, p. 17). Gowers develops and defends in his book what he calls the abstract method in mathematics, which he encapsulates in slogans like "a mathematical object is what it does" (1.c., p. 18), or, less metaphorically, "think about the rules rather than the numbers themselves" (1.c., p. 22). Presumably, Parsons would diagnose in Gowers' case-rightly or wrongly, I do not want to decide this here-a lack of "sensitivity to the difficulties that philosophical positions are prone to", of which he says that it "is the most difficult for a mathematician to achieve" (Parsons 2006, p. 147).

12 How does this fit Parsons' seemingly coherentist picture regarding sets (presented on p. 99), that I mentioned before?-According to Parsons, this picture, too, has to be enriched by what he calls a "bottom for the set theoretic hierarchy", which should be constituted by "more intuitive models" (p. 113). It is these intuitive models instantiating the structure of the natural numbers which he considers to be necessary to solve the problem of nonvacuitiy.
} 
whereas the notion of “apriority" loses the central place it has in Kant's thinking. ${ }^{13}$ In Parsons' thinking also the second one-the function to prevent us from running into antinomies - is downgraded, but the first one-the function to give us objectsgets an especially prominent position. When trying to defend a Kantian concept of "intuition", Parsons' originality lies precisely in the way in which he wants to preserve this first function.

Thus, seeing his structuralist position threatened by the problem of nonvacuity and rejecting physicalism and similar materialist positions which search for the pertinent structures in the physical world, Parsons' way out is to pin his hopes on our intuition of objects like the strings of strokes characteristic of Hilbert's finitary arithmetic, which seem to supply us with what Parsons calls an intuitive model of arithmetic. According to my understanding of his book, this is the essential function of the notion of "intuition" in his philosophy. For Parsons, the existence of the intuitive model of strings of strokes cannot be reasonably disputed, and with this philosophical move he wants to solve the problem of nonvacuity-at least in the case of arithmetic, and this is the paradigmatic case to which I here restrict myself. ${ }^{14}$

Parsons' idea of an 'intuitive model' of arithmetic is very suggestive, and aspects of it can also be found in Hilbert's thinking. However, it provokes severe objections that remain unanswered, and this is what I want to show in the next section.

\section{Intuitive Models}

In his answers to 5 Questions about the philosophy of mathematics, Parsons says that from early on in his career the following question has been important to him: "Had it really been proved, as was often claimed, that logicist and set-theoretic constructions made Kant's pure intuition, or other ideas deriving from it, otiose?" (Hendricks and Leitgeb 2008, p. 206). It was not only the weight of Kant's philosophy that disposed him to take this question seriously, but above all the influence of Hilbert and his prooftheoretic program of the 1920s. I think that Parsons' aims in the philosophy of mathematics can only be adequately understood if one recalls the following famous declaration of Hilbert in "Über das Unendliche" from 1925:

Kant already taught - and indeed it is part and parcel of his doctrine - that mathematics has at its disposal a content secured independently of all logic and hence can never be provided with a foundation by means of logic alone; that is why the efforts of Frege and Dedekind were bound to fail. Rather, as a condition for the use of logical inferences and the performance of logical operations, something must already be given to our faculty of representation [in der

\footnotetext{
${ }^{13}$ Hilbert tries to retain a certain sense of it when at the beginning of Hilbert 1931 he assigns it to his finite mode of thought ("finite Einstellung"), but this is certainly problematic, and it does not play an important role in Hilbert's proof theoretical approach.

14 This reading of Parsons' book may also help to clarify in what way he is no adherent of a sui generis view of natural numbers. For in this case the natural numbers themselves could be referred to as constituting a model of arithmetic and as preventing his structuralism from vacuity. 'Intuitive models' would not be needed.
} 
Vorstellung], certain extra-logical concrete objects that are intuitively [anschaulich] present as immediate experience prior to all thought. If logical inference is to be reliable [sicher], it must be possible to survey these objects completely in all their parts, and the fact that they occur, that they differ from one another, and that they follow each other, or are concatenated, is immediately given intuitively, together with the objects, as something that neither can be reduced to anything else nor requires reduction. This is the basic philosophical position that I consider requisite for mathematics and, in general, for scientific thinking, understanding, and communication. And in mathematics, in particular, what we consider is the concrete signs themselves, whose shape, according to the conception we have adopted, is immediately clear and recognizable. ${ }^{15}$

This is the core of Hilbert's 'finitism'. I think that Hilbert's own main motive for it was not philosophical but intra-mathematical, and also, in a sense, of a pragmatic nature, aiming at the consensus within the community of mathematicians by devising finitary consistency proofs that are acceptable also to intuitionists, such that, in the end, intuitionists might become ready to put up with classical mathematics. After Gödel's incompleteness theorems it would be hard to maintain this sort of intention. In any case, it is not Parsons' intention when relying on 'intuition' in his philosophy of mathematics.

In his usage of the word "intuition" Parsons adopts Hilbert's narrow concept of "Anschauung", which is meant in the sense of an intuition of perceptible objects. In what follows I will concentrate on Hilbert's strings of strokes as the paradigmatic objects that are relevant to mathematics: I, II, III, IIII, .... ${ }^{16}$ This sequence of perceptible signs obviously mimics the sequence of natural numbers, and we have to understand the specific role it may play in mathematics. The domain of these objects then also allows intuition that certain states of affairs obtain, and in this way it allows intuitive knowledge-e.g., that the string IIII is 'greater' (in an obvious intuitive sense of this word) than the string II, etcetera. According to Parsons, a decisive trait of this Hilbertian intuition of objects is its analogy with perception, ${ }^{17}$ an analogy which is evident in the case of strings of strokes.

One must not think, however, that this analogy forces Parsons to consider objects of intuition, like our strings of strokes, really as 'concrete objects' in the strict sense of the term "concrete". This, of course, would from the outset spoil his intention to

\footnotetext{
15 Hilbert 1926, p. 376. This sort of remark goes back to Hilbert 1922, where it is crowned with the exclamation: "In the beginning was the sign [am Anfang ist das Zeichen]" (Hilbert 1922, Sect. 25), and it is repeated almost verbatim in several of Hilbert's papers from 1922 to 1931. Remarkably, in 1922 Hilbert did not talk about 'certain extra-logical concrete objects', but about 'certain extra-logical discrete objects"! I do not know what prompted this change from "discrete" to "concrete" and why Hilbert might have considered it an improvement (shouldn't it be seen conversely?).

16 Strictly speaking, these are not the paradigmatic objects Hilbert himself is talking about. Instead of strings of strokes, Hilbert always uses strings of the sign " 1 " (this is even so in Hilbert and Bernays 1968). I do not know what his reasons were to stick to this presentation, which has certain drawbacks (e.g., Hilbert uses the symbol "1" also as a 'Mitteilungszeichen' referring to the sign " 1 ", which to my mind is a rather awkward decision). As usual today, Parsons uses strings of strokes because of their (as one might say) more elementary shape.

17 "It is hard to see what could make a cognitive relation to objects count as intuition if not some analogy with perception" (p. 144).
} 
be able to treat them as mathematical objects. For him they are abstract types, which he calls "quasi-concrete", because they have a specific, internal relation to their concrete tokens. The official explication of the term "quasi-concrete object" is as follows: "Some abstract objects are distinguished by the fact that they have an intrinsic relation to the concrete; they are determined by their concrete embodiments. I shall call such objects quasi-concrete. [...] What makes an object quasiconcrete is that it is of a kind which goes with an intrinsic, concrete 'representation', such that different objects of the kind in question are distinguishable by having different representations." (pp. 33-34) ${ }^{18}$ In what follows, when referring to a "string of strokes' I always mean it in Parsons' sense of a type of string of strokes, i.e. as a quasi-concrete object. Furthermore, it is quite evident, that also Hilbert meant his 'concrete' objects in a sense like this. ${ }^{19}$ In 1922 he explicitly says that "the objects of number theory are for me [...] the signs themselves, whose shape can be generally and certainly recognized by us-independently of space and time, of the special conditions of the production of the sign, and of significant differences in the finished product", and he adds in a footnote: "In this sense, I call signs of the same shape 'the same sign' for short" (Hilbert 1922, paragraph 25).

In spite of the abstract nature of a string of strokes, we can nevertheless legitimately talk about our perceiving it. I agree with Parsons "that talk of perception of types is something normal and everyday" (p. 159) and that also a philosopher is allowed to accept it as sufficiently understood. At the same time, however, Parsons, following Hilbert, wants to regard these perceptible objects as properly mathematical (p. 151), and here both run into difficulties. Problems arise with respect to the identity of the strings of strokes, i.e. with respect to our talking of the same and not the same type. Parsons presents a particularly characteristic problem of this sort on p. 163:

Consider the examples

IIII
III I
III I
III I
III I.

At what point does the inscription cease to be of the same type as the initial one? They all satisfy an obvious criterion in that they each consist of four strokes. So one might say at no point. But that is not at all plausible; no one would say, for example, that the first of the above strings continued by 'l' one thousand miles to the right constitute a single string of five strokes.

\footnotetext{
18 As noted in Smith 2009, p. 6, the word "representation" is not used here in the semantic sense of referring to the represented object, but in the sense of being representative of it: the concrete token is a representative of the type. However, I will stick to Parsons' terminology.

19 It would be interesting to investigate what, in different circumstances, mathematicians have in mind when using the term "concrete", which is rather often the case.
} 
Silly problems of this caliber abound with respect to strings of strokes (and in case of other quasi-concrete objects as well). ${ }^{20}$ Let us call them vagueness problems, for short. They threaten Hilbert's finitism as well as Parsons' elaboration of it, because they seem to make strings of strokes into vague objects, or to make the concept of a string of strokes into a vague concept, which is not allowed in mathematics. In such a case our intuition of strings of strokes could not be considered as mathematical intuition:

What makes intuition mathematical intuition is that it gives objects that instantiate concepts that have a sharp, precise character. At least for statements in the mathematical vocabulary, there is no vagueness in their application to strings of strokes. There may indeed be vagueness as to whether what is before us, or is not, a token of a given string, but not about the question whether one string, say, consists of two more strokes than another. (pp. 165-166)

Parsons' attempt at counteracting this threat of vagueness consists in two steps. First he rejects the view, suggested in the just mentioned footnote of Hilbert 1922, that a quasi-concrete sign like "IIII" should be identified with an equivalence class of concrete tokens, where the equivalence relation is the relation of being of the same shape. ${ }^{21}$ He calls this view "nominalist" (p. 160), because it involves a reduction of types to their nominalistically more respectable tokens. Instead, Parsons prefers to be more respectful to our actual handling of types and tokens, and he convincingly shows on pp. 164-165 that the nominalist reduction does not accord with our normal ways of identification of types and tokens, which are relatively independent of each other. For Parsons this is also reason not to speak of our perception of types, but rather of our intuition of them:

I do not want to say [...] that seeing a stroke-inscription necessarily counts as intuition of the type it instantiates. One has to approach it with the concept of the type; first of all to have the capacity to recognize other situations either as presenting the same type, or a different one, or as not presenting a string of language at all. But for intuiting a type something more than mere capacity is involved, which, at least in the case of a real inscription, could be described as seeing something as the type. (p. 165)

The relation of a type to its tokens is not a relation of reduction, but one of foundedness (in the sense of Husserl), and according to Parsons it is not only our perception of tokens, but also our imagination, that plays a paradigmatic role in our intuition of types (pp. 161-162). In this way "intuit" generates an intensional context (p. 185) that does not conform to nominalist simplifications.

\footnotetext{
${ }^{20}$ One might think of circumventing these problems by standardizing the strings, for example by using means coming from geometry; by demanding, say, that in one string all the neighboring strokes should have the same distance from one another, and maybe even a specific distance. But such a move is not allowed by Parsons' setting, because 'having the same distance' cannot be seen. For this reason, when presenting what he calls 'intuitive models' of arithmetic, Parsons cannot allow full-blown geometric models.

21 Parsons argues on pp. 241-242 that Hilbert's footnote of 1922 should not be taken seriously and that Hilbert should be interpreted in the way which he himself develops in his book.
} 
Sophisticated as this view may be, it unfortunately does not solve the vagueness problem that gave rise to it, because this problem is also present on the level of types even if these types are not nominalistically reduced. To speak for myself, when being confronted with the picture of the different strings of strokes on p. 163, I automatically see them as types, and it would require an altogether artificial attitude to take them as tokens or even as equivalence classes of tokens. Nonetheless, the vagueness problem remains: why not still "say, for example, that the first of the above strings continued by ' $l$ one thousand miles to the right constitute a single string of five strokes" (p. 163)?

At this point Parsons makes a further step. It consists in the thought that it might be "possible to shunt off any threat of vagueness in their individuation [i.e., in the individuation of types] onto the relation to their tokens or possibly the individuation of the tokens" (p. 168). What Parsons thereby means is this: suppose that the following type is uncontroversial to us (which it certainly is):

(a) IIII

The vagueness that concerns us, however, arises if we ask whether this

(b) III I

is the same type. Parsons' shunting-off-strategy, then, consists in arguing as follows: "our difficulty concerns a relation between a type, the type of (a), and the token of (b). [Footnote: The reader is again asked to take that token to be the particular inscription marked (b) in the particular copy of this work that he has at hand.] That relation can be vague without the relations between types becoming vague. The vagueness may generate ambiguity as to what type 'the type instantiated by (b)' designates (if we do not take it to fail of reference because of failure of uniqueness). But if we admit that it does designate a type, then the identity statement the type instantiated by (a) = the type instantiated by (b) cannot be vague, although it may be ambiguous" (pp. 168-169).

This answer, however, is nothing but hand-waving as long as Parsons does not tell us anything about the way in which the token of (b) is becoming the token of a certain type (of the same type as IIII, say), or of two types (III and I, say), or of none. If I see it correctly, Parsons remains totally silent concerning this crucial issue. One might consider the reply that at precisely this point the notion of a type and, correspondingly, the notion of a quasi-concrete object turn out to be theoretical concepts, such that the question of the (b)-token's 'becoming' a specific type need not be answered ${ }^{22}$; but this reply would appear ad hoc, and it would contradict the descriptive approach that Parsons has in mind. In any case, Parsons himself does not give such a reply, and on p. 169 he in fact admits to have merely shown that vagueness phenomena like the one presented on p. 163 do not force us to admit "that the notions of stroke, string of strokes, or identity applied to such strings are vague", and this is an extremely weak result. Its weakness is confirmed on pp. 246-247, where Parsons says that "readers may legitimately feel that more should be said about equality of strings". But he then

\footnotetext{
${ }^{22}$ I am grateful to Wilfried Keller for suggesting this sort of reply to me.
} 
only adds two principles relevant to our understanding of strings - saying that two strings each consisting of a single symbol are equal, and that equal strings have equal successors - which have nothing at all to do with 'intuition'.

It seems to me that Parsons' difficulties at this point stem from an excessive fixation on the objects themselves - the types I, II, III, ...- -and a far-reaching neglect of the rules according to which we produce and use these objects. Parsons seems to think that talking about our intuition of these objects can replace talk about rules and use, but his reflections make rather clear how hopeless it is to try to say something substantial about the identity of the objects without taking our rulegoverned use of them into consideration. There are two sorts of rules which are relevant in the present context. Roughly speaking, they correspond to the role of natural numbers as ordinals or as cardinals. Their ordinal role is expressed by the following rule of production:

(O) Start with the object I, then add this same object, then again this object, and so on; at each step first reproduce the whole object produced at the step before and only then add I.

Obviously, this rule gives rise to the well-ordering I, II, III, ..; but, strictly speaking, the structure which it produces is much richer than an order structure (also, say: I, $\||||$,$| , IIIIIII, III, etc., could be regarded as such a well-ordering, as long as the objects$ turning up in this way are counted as different objects), and it immediately leads us to the role of these strings as cardinals, governed by the following rule:

(C) In order to know whether a given collection of objects has the cardinality IIIII (called "five", for short), try to put the objects in one-one-correspondence with the strokes of IIII; if this is possible, then the collection does have said cardinality, if it is not possible, it doesn't.

Obviously, this rule can be refined, and it leads to multifarious further uses of the strings of strokes, which need not be described here. Its essence lies in giving the strings of strokes the role of standards of numbers. ${ }^{23}$

This rule-oriented perspective allows a very natural treatment of our vagueness problem concerning the identity of strings of strokes: In the context of a person following rule $(\mathrm{O})$, the token

(b) III I

of p. 168 should be regarded as of the same type as

(a) IIII,

if the person treats (b) as occurring immediately after III and immediately before IIII. And in the context of a person following rule (C), the token (b) should be regarded as of the same type as (a) if the person treats (b) as one number-standard and not as two or as none. From a perspective like this, Parsons' verdict on p. 163, that "no one would say, for example, that [the string (a)] continued by 'I' a thousand miles to the

$\overline{23}$ This role is beautifully described in Mazur 2007, pp. 4-5. 
right constitute a single string of five strokes", appears infelicitous, because just this could very well be said if one had the corresponding use in mind; for example the use of a standard of fiveness in a situation where the collection of objects that we want to measure by this standard, itself shows such an extremely dispersed distribution. (Of course, this is a rather fictitious example, but the point to be made here is one of principle.)

This is the way in which the identity of the signs I, II, III, ... is determined. It is not a precise mathematical determination, but a context-dependent one, and it obviously breaks down when the strings become too long. ${ }^{24}$ It is so different from the way in which we deal with the identity of the natural numbers, that we should refrain from regarding the objects I, II, III, ... as mathematical objects. Not only the way in which we settle the identity of these objects, but also the way in which we refer to them is all too different from the case of natural numbers. Our reference to the I, $\|\| I,, \ldots$ is governed by rules like $(\mathrm{O})$ and $(\mathrm{C})$, which are rules explicitly concerned with our actions, and this leads to a characteristic context-dependence and restrictedness to short strings in our way of referring to them. In contrast to this, the way in which we treat the identity of the numbers themselves and in which we refer to numbers is mainly governed by the acceptance of mathematical propositions-axioms, equations, theorems-and in this way we transcend, so to speak, the contextdependence and restrictedness characteristic in the case of the I, II, II, ... .

As already mentioned, Parsons opposes a narrow-minded "legislation about what counts as a mathematical object" (p. 116), and in principle I agree with his liberal attitude. But the objects I, II, III, ... seem to be clearly on the non-mathematical side. Consequently, Parsons' idea to consider the domain of these objects as an intuitive model of arithmetic is inappropriate. If I understand Parsons correctly, models should be and should consist of mathematical objects-but according to the ruleoriented view just developed, the property of being an object of intuition is inconsistent with this. Seen in this way, Parsons' talk of 'intuitive models' looks like a category mistake.

It is a mistake we all are prone to, I think. How is it to be explained? How could it come about?-The main reason might be that we understand what natural numbers are, and how to deal with them, and we accept the sort of precision and sharpness that reigns in the domain of natural numbers, long before we get acquainted with the Hilbertian 'visual numbers' I, II, III, .... And when we then meet this domain of visual numbers, we automatically project our antecedent understanding of numbers into it and measure what is happening in it against what we know from the numbers we are familiar with. In this way we unconsciously also project the latter's sharpness and precision into it.

One can express this tendency also in another, more Wittgensteinian manner: evidently, pictures and signs (considered as visual shapes) as such do not contain their own rules of use-something must be additionally present, so to speak. What is this something? According to Wittgenstein, it is the use itself in form of a certain

\footnotetext{
${ }^{24}$ The relevance of this sort of breakdown is investigated in Wittgenstein's reflections on the 'surveyability' of proofs in Part III of his Remarks on the Foundations of Mathematics; see my attempt at clarifying this topic in Mühlhölzer 2005.
} 
established practice. In his Blue Book he introduces the beautiful metaphor of the life of the sign which is bestowed on the sign by its being used within a certain linguistic practice, and Wittgenstein's view is that anything else which we might add to a sign is, in the end, at best just another sign. ${ }^{25}$ Parsons' discussions, on the other hand, often give the impression that he wants to assign just this life-giving role to our intuition, at least in the case of the signs I, II, III, ... But I do not see that 'intuition' can take on this role. What is important is not what the signs I, II, II, ... look like, but what we do with them. Their appearance may be psychologically relevant to us in our practice of dealing with them, but in the end it is the practice itself, quite independently of the psychological conditions on which we actually depend, that should be seen as semantically and epistemically important.

I think that precisely at this point Parsons' philosophy of mathematics has serious gaps, and to my mind this is particularly obvious in his investigation of the limits of intuitive arithmetic in Chap. 7. Again, it is the arithmetic with the Hilbertian 'numbers' I, II, III, ... which he takes as his paradigm of an 'intuitive arithmetic'. In $\S$ 44 of this chapter, Parsons develops and defends the view that, although addition, multiplication and exponentiation are alike in being primitive recursive functions, only the first two belong to intuitive arithmetic, whereas exponentiation goes beyond the domain of what is intuitively accessible. ${ }^{26}$ The characteristic fallacy that I would diagnose at this point, however, lies in the (unconscious, implicit) assumption that in the case of addition and multiplication intuition as such shows us a certain determinateness of these functions which guarantees that they are welldefined, whereas in the case of exponentiation we do not have a guarantee of this sort.

Let me explain this in more detail: All three functions can be characterized by recursive definitions of the following form:

(R) $f(1)=a$

$$
f(n+1)=g(f(n), n),
$$

where $g$ is a function already given and accepted, and $f$ is the function introduced by giving its value for the argument 1 and by specifying a procedure for the subsequent step-by-step calculation of $f(2), f(3), \ldots$ through an iterated application of $g$ (thereby mimicking, in this iteration of the application of $g$, the iteration of the application of the successor function characteristic of the development of the sequence of the natural numbers itself). Iterations, however, are nothing that Parsons would consider as 'intuitive' in the sense relevant to his 'intuitive arithmetic', and therefore he demands intuitive arguments for the well-defined character of functions

\footnotetext{
25 Wittgenstein 1969, pp. 4-5. I say more to this Wittgensteinian metaphor in Mühlhölzer 2008 and 2010.

26 With this claim Parsons puts himself in opposition to William Tait's well-known analysis of 'finitism' in Tait 1981. Tait defends the view that finitism should not be characterized via 'intuition', but as the position that only allows the reference to finite entities. Tait then comes to the conclusion that finitist arithmetic is identical with primitive recursive arithmetic. Parsons, on the other hand, wants to defend a version of finitism - which he now calls "intuitive arithmetic" — based on 'intuition of objects', and his result is that this arithmetic is considerably narrower than primitive recursive arithmetic. (See also Parsons' discussion of finitism in Sect. 4 of Parsons 2009.).
} 
defined according to (R). ${ }^{27}$ That is, he wants to show in an intuitively satisfactory way that, given a step-by-step procedure of type (R), for every $n$ there exists a determinate $k$ such that $k=f(n)$. But from the point of view of intuitive arithmetic, such an existential quantification should "be cashable by explicitly giving the instance making the statement true" (as Parsons says on p. 258). ${ }^{28}$ To give such an instance, however, seems to involve just a calculation according to (R) - and we land in a circle: in order to prove that the procedure of type $(\mathrm{R})$ is well-defined, we already presuppose that it is.

According to Parsons, this circle can be circumvented in the case of addition and multiplication, because there we can present definitions that do not rely on iterations as put down in (R), in contrast to exponentiation, where iteration cannot be avoided. For addition, Parsons presents the intuitive idea of concatenation of strings, that is, of an intuitive procedure visualized by something like this:

(A) IIIIII concatenated with IIIII yields IIIIIIIIII,

which corresponds to " $7+5=12$ ". For multiplication, the intuitive idea is the idea of a replacement of strokes by strings in the following way:

(M) Each stroke of III replaced by I||| yields IIIIIIIIIII,

which corresponds to " $3 \cdot 4=12$ ". If I understand Parsons correctly, he wants to say that these intuitive ideas show, in a way that is independent of the idea of iteration, that addition and multiplication are well-defined functions, with the result that these two functions belong to intuitive arithmetic. On the other hand, it is not to be seen how something analogous could be accomplished in the case of exponentiationwhence exponentiation is excluded from intuitive arithmetic.

It seems to me, however, that this argument is based on some sort of delusion. Certainly, our intuition of the strings of strokes alone, and of pictures like (A) and (M) alone, is not sufficient to define a determinate function. These strings and these pictures, considered for themselves, are dead signs (to use this Wittgensteinian metaphor again), and Parsons' 'intuition' can no more bring them to life than anything else can; that is: it cannot determine what they are supposed to mean. By simply looking at the signs and the pictures, their meaning does not disclose itself. Here we immediately run into the Wittgensteinian rule-following issue. If one finds Wittgenstein's reflections concerning rule-following convincing (as I do), then one should say that this meaning must be materialized, so to speak, in our actions, and that the determinateness of the functions, which Parsons is concerned about, has to be sought in the way we actually deal with the functions and not in 'intuitions'. The way in which we treat this determinateness issue has to be sought in our mathematical practice, and in the light of this practice the difference between addition and multiplication, on the one hand, and exponentiation, on the other, does

\footnotetext{
${ }^{27}$ In this demand lies the essential difference to Tait's approach. For Tait, a recursive definition like (R) describes a construction of the values of $f$, based on the fundamental idea of iteration, and for him it makes no sense to ask for a proof that a procedure of this sort is well-defined (see Tait 1981, pp. 27-28). For this reason, Tait does not run into the circle bothering Parsons, which we will come across in a moment.

28 This, of course, is also true for the finitist view defended in Tait 1981.
} 
not appear as deep as Parsons suggests. His reflections simply do not tell us enough about our practice in order to present a satisfying view of the role 'intuition' might play in it. This role remains all too nebulous.

Parsons' himself seems to feel that his reliance on 'intuition' is too weak at this point, because he seeks support through a different argument making use of the notion of 'feasibility' in the sense of polynomial-time computability. In this sense, addition and multiplication are feasible, whereas exponentiation is not (see p. 256). This is in fact a clear distinction, but it has nothing to do with the role of intuition in guaranteeing the well-definedness of the functions.

What, then, is the role of intuition? As already said, what I clearly see is a psychological role-certainly, it is easier for us to use signs such as strings of strokes than more irregular ones-but not the deep systematic role Parsons wants to ascribe to it. Furthermore, intuition becomes important in our extra-mathematical application of arithmetic; for example in our application to the signs I, II, II, ... themselves! When applying arithmetic to such objects, the identity of these objects is given in advance; whereas the numbers, which arithmetic is about, are only given by arithmetic itself. There is obviously a deep difference between application and aboutness, and it seems to me that the proper way to look at the signs I, II, III, ... is to treat them simply as objects of the application of arithmetic, and not as arithmetical objects themselves. ${ }^{29}$

What complicates the situation, however, is the fact that the strings of strokes can also be used as signs of arithmetic. If we think of our practice as properly enriched by treating these strings like singular terms, by introducing predicates and function symbols like "=", “<", "+" etc., and by providing appropriate means for quantification, such that this practice becomes sufficiently similar to our familiar mathematical practice, then we should be allowed to regard the strings of strokes as signs with meaning and with reference to objects. ${ }^{30}$ These objects, however, are now the natural numbers themselves. We then have the familiar two levels of (i) the signs, used in a certain, meaning-bestowing way and subject to their own nonprecise criteria of identity, and (ii) the precise mathematical objects referred to by these signs.

Incidentally, it should be noted that if we use the strings of strokes as numerals, i.e., if we $d o$ arithmetic with them, and if at the same time we bear in mind that they are also objects of the application of arithmetic, we can pointedly say, as Wittgenstein did in a notorious passage of his Big Typescript, that "arithmetic is its own application" (Wittgenstein 2005, p. 550).

\footnotetext{
29 This application concerns strings of strokes as types and not only strings of strokes as tokens. I completely agree with Parsons' endeavor to develop a descriptive approach towards types and our intuition of types, which makes it abundantly clear that it is types that we normally have in mind when considering strings of strokes, and therefore it is types as well when we apply arithmetic to strings of strokes.

30 I am reluctant to say that Parsons himself has presented criteria for meaning, because "meaning" is a subject which is conspicuously absent in his book. In his paper "Quine and Gödel on Analyticity", Parsons observes that Gödel, in contrast to Quine, never really got into deeper discussions about meaning (Parsons 1995, p. 309). Almost the same, however, is true of his own philosophy of mathematics as developed in his book.
} 
When using the strings of strokes as numerals, we veer away from Hilbert's view of finitary arithmetic. According to Hilbert, the strings of strokes are not signs with meaning and reference; Hilbert does not allow sentences with them like "II $<$ III" or "II +\|\|$=\|\| \| "$; and therefore the strings of strokes are not signs belonging to a language. Hilbert expresses the sentences just intended as " $2<3$ " and " $2+3=5 "$ on the level of what he calls signs for communication, ${ }^{31}$ with our familiar numerals "1", "2", " 3 " etc. as signs referring to the objects I, II, III etc. Nothing could be easier, however, than to use also the strings of strokes as numerals referring to our familiar natural numbers. And our familiar numerals in decimal notation, which refer to these numbers as well, then appear simply as notational variants, possibly as abbreviations of strings of strokes.

From this perspective we may get a still better understanding of our tendency to consider the strings of strokes as mathematical objects. I think we have a tendency to automatically read them as numerals referring to numbers, and then we retroactively (so to speak), but unconsciously, use these numbers as norms for the identity of the numerals themselves. There is a certain indication that precisely this is what happens in Parsons' case, for Parsons-quite uncharacteristically in view of his usual scrupulousness - at one place in his book, ${ }^{32}$ and in a context where he explicitly claims to be following the Hilbert school, ${ }^{33}$ writes down formulae like " $\|=\|$ " and " $\|=\|\|\rightarrow=\| "$ ", without any warning that this contradicts Hilbert's intentions. He here uses the strings of strokes as numerals, and he may have unconsciously projected onto these numerals essential features of the numbers which they refer to.

So Parsons' idea to rescue his structuralism from vacuity by considering the strings of strokes as mathematical objects constituting an 'intuitive model' of arithmetic provokes too many objections that are left unanswered in his book. However, this does not alter the fact that 'intuitive knowledge' simply belongs to our mathematical practice and experience, and that it may be worthy of being recognized in itself. Hilbert himself was impressed by this very fact, and we should come to an adequate understanding of it independently of Parsons' systematic ambitions with respect to his structuralism.

\section{Intuitive Knowledge}

Parsons' discussion of primitive recursive functions, which I presented and criticized in the foregoing section, actually does not belong to this section's topic, "Intuitive Models", but has its place in Parsons' Chap. 7 ("Intuitive arithmetic and its limits") which deals with our intuitive knowledge of arithmetical truths. Thus, Parsons' result concerning the non-intuitive character of exponentiation is presented by him as a result concerning our knowledge of the well-definedness of the exponential function. Because to him it is not intuitively evident that in this case the

\footnotetext{
31 "Zeichen zur Mitteilung"; see Hilbert and Bernays 1968, p. 21.

32 On p. 173.

33 See p. 172.
} 
computation terminates for all arguments, Parsons is "inclined to the negative conclusion, that the arithmetic that is intuitively known does not include exponentiation" (p. 260). When, in the foregoing section, I raised doubts concerning Parsons' idea of an 'intuitive model' of arithmetic, this need not necessarily affect Parsons' conviction that intuitive knowledge-in the Kant-Hilbert sense of "intuitive"-might play an important role for our understanding of mathematics, and we should now clarify this role. In his paper "Intuition in constructive mathematics" Parsons states that "on both Brouwer's and Hilbert's views, intuition in a roughly Kantian sense is a basic and indispensable source of mathematical knowledge" (Parsons 1986, p. 211), and his own aim is to fathom views of this sort in order to defend a certain robust core of them.

What is 'intuitive knowledge' in Parsons' sense? At the beginning of $\S 29$ he says that "an item of intuitive knowledge would be something that can be 'seen' to be true on the basis of intuiting objects that it is about", like our seeing it to be true that III is the successor of II. But of course this account has to be generalized in order to allow intuitive knowledge also of general truths, like the truth that the successor function is a well defined total function, or that laws like the commutative law of addition are true. ${ }^{34}$ To this end Parsons allows our intuiting of arbitrary strings of strokes, and he allows certain inferences which count as preserving intuitive knowledge. The reasons which he gives for these sorts of extensions of the original idea of an intuition of particular objects are, to my mind, rather convincing. In all these cases intuitive knowledge is knowledge of propositions which we judge as 'intuitively evident'. That is the terminology which Parsons uses in this area.

I would like to end this paper with a very short discussion of the case of simple statements like

(1) $7+5=12$,

which Parsons considers as "intuitively evident when interpreted with reference to an intuitive model" (p. 224), where the intuitive model he has in mind is always the model of strings of strokes. That is to say, what he has in mind here is the following reading of (1):

(2) IIIIIII concatenated with IIII| yields IIIIIIIIII.

Let us ask in which way (1) might appear intuitively evident when seen in the light of (2).

In order to answer this question, one should note, first, that (2) tells us something about (1) only when we recognize what numbers are involved in (2): that it is really the numbers 7,5 and $12 .{ }^{35}$ How do we recognize this? In the case of IIII one might see

\footnotetext{
34 As already mentioned, Hilbert gives a nice intuitive proof of this in Hilbert 1922, paragraph 30.

35 This beautiful question, which at first sight—but only at first sight—sounds so harmless, is raised and discussed in Wittgenstein 1978, Part III, §§ 6-8, by which the following reflections are prompted. They are also prompted by a seminar about this question given by Saul Kripke in September 1998 at Humboldt University, Berlin, entitled "Logicism, Wittgenstein, and the Identification of Numbers", in which Kripke presented and expanded thoughts of his Whitehead Lectures at Harvard University, "Logicism, Wittgenstein, and de re Beliefs about Numbers", of 1992.
} 
immediately what number it is-but for IIIIII this is more difficult, and it is definitely not so (at least to me) for I||||||||||. In the latter case I have to count the strokes: $1,2,3, \ldots$, 12-and a process like this is unavoidable for all strings that are longer. But this means that, if the numbers involved are not very small, our insight into truths like (1) needs much more than merely (2). And if we take a realistic look at (1) we have to admit that we actually recognize its truth via our understanding of decimal numbers and of the usual process of adding such numbers-images of strings of strokes do not play any role (at least in my own thinking). One might ask in what way 'intuition' comes into play in our understanding of decimal numbers and of the usual operations with them. This, however, is rather unclear to me, and I shall not pursue this issue here because it would take us away from Parsons' thoughts.

Of course, we can concentrate on (2) alone, without relating it to (1). Is (2), when considered in itself, intuitively evident? Can we 'see' its truth (as Parsons demands)?-Certainly not without further ado. It is not necessary to count the strokes, but we nevertheless need certain auxiliary means to recognize that there are as many strokes in |||||| $\mid$ and |||| $\mid$, taken together, as in |||||||||||| . For example, we can draw curves connecting the strokes and thereby verify the equinumerosity. To give us a chance to really see the truth of (2), the curves must be drawn in an orderly fashion (which the reader may produce for himself). However, I am still not totally sure that I can 'see' the equinumerosity in pictures with such curves (they flicker before my eyes and I am still tempted to count the strokes in order to play safe), and this insecurity grows with growing length of the strings. I am unsure in these cases whether the curves are drawn correctly.

But what does "correctly" mean here? What and where is the standard of correctness in these cases? It cannot merely lie in a further picture, because a picture in itself can only give such a standard if it is understood in a certain way-and we again run into the Wittgensteinian rule-following issue. According to Wittgenstein's reflections concerning rule-following this understanding must be manifested in our actions, and the standards of correctness have to be sought in these actions as well. That is, they must be sought, as Wittgenstein writes in \$201 of Philosophical Investigations, in our characteristic "way of grasping a rule [...] which is exhibited in what we call 'obeying the rule' and 'going against it' in actual cases". If seen in that way, one had better refrain from talking of the 'intuitive evidence' of (1) and (2), or of our 'seeing the truth' of (1) and (2), because this truth is to be judged according to the correctness standards exhibited in our use of the signs. 'Intuitions' (in Parsons' sense of this term) cannot play the role of such standards. To treat them in such a way would be a philosophical fiction. ${ }^{36}$ If, upon Wittgenstein's advice, we look at our actual practice of judging the correctness of mathematical statements, we find that 'intuitive' standards like IIII are only used, if at all, for very short strings (that III concatenated with II yields IIII may pass as intuitive standard for " $3+2=5 ")$; that we use notational systems like the decimal notation in order to be able to calculate that, say, 3726 plus 1217 equals 4943; and that we invent new

\footnotetext{
36 This sort of fiction may also be diagnosed in Kant's conception of 'pure intuition'. I argue for this in Mühlhölzer 2002.
} 
notations, like exponentiation, super-exponentiation, and so on, ${ }^{37}$ which allow reliable correctness judgments in case of ever greater numbers. It is a phenomenon like this that Wittgenstein has in mind when he speaks of the "way of grasping a rule [...] which is exhibited in what we call 'obeying the rule' and 'going against it' in actual cases". There is, if at all, only an extremely humble role that 'intuition' might play here.

All this is epitomized in Wittgenstein's wonderful remark: "Nicht, dass er uns als wahr einleuchtet, sondern dass wir das Einleuchten gelten lassen, macht ihn zum mathematischen Satz." 38 Wittgenstein's term "gelten lassen" refers to our actual practice of judging whether a mathematical statement is correct, and it is not our finding the statement 'als wahr einleuchtend' (i.e.: our finding it 'self-evidently true'), but our 'gelten lassen' (i.e.: our actual 'judging it true') that makes it into a mathematical proposition expressing mathematical knowledge. ${ }^{39}$

What, then, $i$ s the role of our finding things self-evidently true, and especially of finding them intuitively evident?-This should be investigated in detail. I cannot do this here, but I think that, in any case, the answers given by Parsons to this question are not convincing. As argued above, strings of strokes, considered as objects of intuition, cannot supply us with a 'model' of arithmetic, and these strings are not suited to playing an important role in explaining our evidence for statements like "7 $+5=12$ ", not to mention more complicated and more demanding cases. It is a characteristic trait of philosophical discussions about 'intuition' that the precise role of "intuition" regularly turns out to be utterly elusive, and Parsons' reflections meet with the same fate.

So the end result of this critical discussion is a negative one. Nevertheless, Parsons' book, the embodiment of his thinking of the past 30 years, is an important achievement, not only because of the many valuable topics and results discussed and achieved in it which this critical discussion left out, but also in view of his probing search after a viable role of "intuition". Irrespective of its success, this search advances into depths that are without precedent. The explorative spirit in which this book is written is a paradigm of formidable philosophy, and if, in the end, the book brings to light more questions than it can answer, this is not the worst thing that might be said about a philosophical investigation. ${ }^{40}$

\footnotetext{
37 As presented, for example, in Boolos et al. 2007, pp. 66-67.

38 Wittgenstein 1984, p. 224. Elizabeth Anscombe's English translation reads as follows: "It is not our finding the proposition self-evidently true, but our making the self-evidence count, that makes it into a mathematical proposition." (Wittgenstein 1978, p. 224).

39 Wittgenstein has his own view of 'mathematical knowledge' which, however, in the present context may be ignored.

${ }^{40}$ I am grateful to Charles Parsons for supplying me with some general orientation concerning the aims of his philosophizing about mathematics, and to Simon Friederich, Wilfried Keller, Bernd Ludwig and Richard Tieszen for reading and criticizing a preliminary version of this paper. I also thank an anonymous referee of this journal for giving me good advice. Furthermore, I profited from discussions with participants of the 'Reflektorium' of the Philosophisches Seminar in Göttingen on May 6, 2009, and with participants of the workshop "The Imaginary, the Ideal and the Infinite in Mathematics" at Pont-àMousson, June 24 to June 27, 2009, organized by Michael Detlefsen and Godehard Link, where I presented preliminary versions of parts of this paper.
} 
Open Access This article is distributed under the terms of the Creative Commons Attribution Noncommercial License which permits any noncommercial use, distribution, and reproduction in any medium, provided the original author(s) and source are credited.

\section{References}

Boolos, G. S., Burgess, J. P., \& Jeffrey, R. C. (2007). Computability and Logic (5th ed.). Cambridge: Cambridge University Press.

Burge, T. (2003). Logic and analyticity. Grazer Philosophische Studien, 66, 199-249.

Burgess, J. P. (2008). Critical study/book review of Charles Parsons'. Mathematical thought and its objects. Philosophia Mathematica (III), 16, 402-420.

Friederich, S. (2010). Structuralism and meta-mathematics. Erkenntnis. doi:10.1007/s10670-010-9210-x. Gowers, T. (2002). Mathematics: A very short introduction. Oxford: Oxford University Press.

Grzegorczyk, A. (2005). Undecidability without arithmetization. Studia Logica, 79, 163-230.

Hendricks, V. F., \& Leitgeb, H. (Eds.) (2008). Philosophy of mathematics: 5 questions. NewYork: Automatic Press/VIP.

Hilbert, D. (1922). Neubegründung der Mathematik. In Abhandl. aus dem Math. Seminar d. Hamb. Univ., Bd. 1, pp. 157-177 (Also in D. Hilbert, Gesammelte Abhandlungen. Band III, J. Springer, 1935, pp. 157-177; and in Hilbertiana, Wissenschaftliche Buchgesellschaft, 1964, pp. 12-32; English translation by W. Ewald in Ewald 1996, pp. 1117-1134; cited according to this edition, using the paragraph numbers added by Ewald).

Hilbert, D. (1926). Über das Unendliche. Mathematische Annalen 95, pp. 161-190 (Also in Hilbertiana, Wissenschaftliche Buchgesellschaft, 1964, pp. 79-108; English translation by S. Bauer-Mengelberg in van Heijenoort 1967, pp. 369-392; cited according to this edition).

Hilbert, D. (1931). Die Grundlegung der elementaren Zahlenlehre. Mathematische Annalen 104, 485-494 (English translation by W. Ewald in Ewald 1996, pp. 1117-1134; cited according to this edition, using the paragraph numbers added by Ewald).

Hilbert, D., \& Bernays, P. (1968). Grundlagen der Mathematik I. Berlin: Springer.

Leitgeb, H., \& Ladyman, J. (2008). Criteria of identity and structuralist ontology. Philosophia Mathematika (III), 16, 388-396.

Mazur, B. (2007). When is one thing equal to some other thing? Ms, June 12, 2007; http:// www.math.harvard.edu/ mazur/.

Mühlhölzer, F. (2002). Regelfolgen und die Identität von Begriffen. In U. Baltzer \& G. Schönrich (Eds.), Institutionalität und Regelfolgen (pp. 137-156). Paderborn: Mentis Verlag.

Mühlhölzer, F. (2005). »A mathematical proof must be surveyable«-What Wittgenstein meant by this and what it implies. Grazer Philosophische Studien, 71, 57-86.

Mühlhölzer, F. (2008). Wittgenstein und der Formalismus. In M. Kroß (Ed.), »Ein Netz von Normen«: Wittgenstein und die Mathematik (pp. 107-148). Berlin: Parerga Verlag.

Mühlhölzer, F. (2010). Wittgenstein and Metamathematics. In P. Stekeler-Weithofer (Ed.), Wittgenstein: Philosophie und Wissenschaften. Hamburg: Verlag Felix Meiner.

Parsons, C. (1986). Intuition in constructive mathematics. In J. Butterfield (Ed.), Language, mind, and logic (pp. 211-229). Cambridge: Cambridge University Press.

Parsons, C. (1995). Quine and Gödel on analyticity. In P. Leonardo \& M. Santambrogio (Eds.), On Quine: New essays (pp. 192-313). Cambridge: Cambridge University Press.

Parsons, C. (2006). Paul Bernays' later philosophy of mathematics. In C. Dimitracopoulos, L. Newelski, D. Norman, \& J. R. Steel (Eds.), Logic colloquium 2005. Lecture notes in Logic 28 (pp. 129-150). Cambridge: Association for Symbolic Logic and Cambridge University Press.

Parsons, C. (2008). Contribution to Hendricks/Leitgeb, pp. 205-210.

Parsons, C. (2009). Critical study/book review of William Tait's. The provenance of pure reason: Essays in the philosophy of mathematics and its history. Philosophia Mathematica (III), 17, 220-247.

Resnik, M. (1981). Mathematics as a science of patterns. Nô̂s, 15, 529-550.

Smith, P. (2009). Critical discussion of Charles Parsons'. Mathematical Thought and Its Objects, Ms, February 2, 2009; http://www.phil.cam.ac.uk/teaching_staff/Smith/logicmatters/Resources/ Parsons1.pdf.

Tait, W. (1981). Finitism. Journal of Philosophy 78, 524-556 (Reprinted in Tait 2005, pp. 21-42; cited according to this edition). 
Tait, W. (2005). The provenance of pure reason: Essays in the philosophy of mathematics and its history. Oxford: Oxford University Press.

Weil, A. (1940). A 1940 letter of André Weil on analogy in mathematics (M. H. Krieger, Trans.). Notices of the American Mathematical Society 52 (2005) 334-341.

Wittgenstein, L. (1958). Philosophical Investigations. In G. E. M. Anscombe \& R. Rhees (Eds.) (G. E. M. Anscombe, Trans.). New York: Blackwell.

Wittgenstein, L. (1969). The blue and brown books. New York: Blackwell.

Wittgenstein, L. (1978). Remarks on the Foundations of Mathematics. (3rd ed.). In G. H. von Wright, R. Rhees \& G. E. M. Anscombe (Eds.) (G. E. M. Anscombe, Trans.). New York: Blackwell.

Wittgenstein, L. (1984). Bemerkungen über die Grundlagen der Mathematik (Werkausgabe Band 6). In G. E. M. Anscombe, R. Rhees, \& G. H. von Wright (Eds.). Frankfurt: Suhrkamp.

Wittgenstein, L. (2005). The Big Typescript: TS 213. German-english scholars' edition (C. G. Luckhardt \& M. A. E. Aue, Ed. \& Trans.). New York: Blackwell. Cited according to the original pagination in TS 213. 